\title{
Rural-Urban Differences in Meeting Physical Activity Recommendations and Health Status in Cancer Survivors in Central Pennsylvania
}

\author{
Scherezade K. Mama, DrPH ${ }^{1,2}$, Nishat Bhuiyan, MS ${ }^{1}$, Wayne Foo, MPH $^{2,3}$, Joel E. Segel, \\ $\mathbf{P h D}^{2,3,4}$, Shirley M. Bluethmann, PhD, MPH ${ }^{2,3}$, Renate M. Winkels, PhD $^{2,5}$, Joachim \\ Wiskemann, PhD ${ }^{2,3}$, William Calo, JD, PhD ${ }^{2,3}$, Eugene J. Lengerich, VMD, MS ${ }^{2,3}$, Kathryn $\mathbf{H}$. \\ Schmitz, PhD, FACSM 2,3
}

${ }^{1}$ Department of Kinesiology, The Pennsylvania State University, University Park, Pennsylvania ${ }^{2}$ Penn State Cancer Institute, Hershey, Pennsylvania ${ }^{3}$ Department of Public Health Sciences, The Pennsylvania State University College of Medicine, Hershey, Pennsylvania ${ }^{4}$ Department of Health Policy and Administration, The Pennsylvania State University, University Park, Pennsylvania ${ }^{5}$ Human Nutrition \& Health, Wageningen University \& Research, Wageningen, The Netherlands

\begin{abstract}
Purpose-This study explored rural-urban differences in meeting physical activity (PA) recommendations and health status in cancer survivors in central Pennsylvania and associations between PA and health status.
\end{abstract}

Methods-Cancer survivors $(N=2,463)$ were identified through a state cancer registry and mailed questionnaires assessing PA and health status. Rural-urban residence was based on county of residence at diagnosis. Participants self-reported frequency and duration of leisure-time PA and were classified as: 1) meeting aerobic recommendations ( $\geq 150$ minutes/week), 2) musclestrengthening recommendations ( $\geq 2$ times/week), 3) both aerobic and muscle-strengthening recommendations, or 4) neither recommendation. Logistic regression models examined associations between rural-urban residence and meeting PA recommendations and associations between PA and health status, adjusting for cancer type, gender and income.

Results-Nearly $600(N=591,24.0 \%)$ cancer survivors returned completed questionnaires (rural $9.5 \%$, urban $90.5 \%$ ). Half (50.0\%) of rural cancer survivors reported no leisure-time PA compared to $35.2 \%$ of urban cancer survivors ( $p=.020$ ), and urban cancer survivors were 2.6 times more likely to meet aerobic PA recommendations (95\% CI: 1.1-6.4). Odds of reporting good physical and mental health were 2.3 times higher among survivors who reported meeting aerobic recommendations compared to those who did not meet PA recommendations (95\% CI: 1.1-4.5), adjusting for rurality and covariates.

Corresponding Author: Scherezade K. Mama, DrPH, Assistant Professor of Kinesiology and Public Health Sciences, Department of Kinesiology, The Pennsylvania State University; 268J Recreation Building, University Park, Pennsylvania 16802, USA; Phone: 1-814-863-5887; Fax: 1-814-865-1275; skmama@psu.edu.

Conflict of Interest: The authors declare that they have no conflicts of interest. 
Conclusions-Results demonstrate persistent rural-urban differences in meeting PA recommendations in cancer survivors and its association with self-reported health.

Implications for Cancer Survivors-Findings underscore the need for interventions to increase PA in rural cancer survivors in an effort to improve health status and reduce cancer health disparities.

\section{Keywords}

Exercise; health behavior; rural health; rural population; health status disparities; cancer survivors

\section{BACKGROUND}

Nearly $20 \%$ of the U.S. population resides in a nonmetropolitan or rural area [1], putting estimates of the number of rural cancer survivors at over 3.1 million [2]. Rural residents have an $8 \%$ higher cancer mortality rate than those in urban areas and experience poorer survivorship outcomes post-treatment [3]. When compared with cancer survivors residing in urban areas, rural cancer survivors are more likely to report two or more non-cancer comorbidities, fair or poor health, poor mental health, increased psychological distress, and unemployment and financial strain due to health problems [4]. Therefore, addressing rural health disparities across the cancer control continuum has emerged as an important public health priority, as supported by recent position statements from the American Association for Cancer Research, the American Cancer Society, the American Society of Clinical Oncology, and the National Cancer Institute calling for an investment in rural cancer control $[3,5]$.

Disparities in health behaviors, such as physical activity (PA), may partially explain cancer health disparities in rural cancer survivors compared with urban cancer survivors. PA reduces the risk of cancer recurrence as well as the risk of developing comorbidities in cancer survivors [6-9]. PA can also improve physical and psychological health and wellbeing [6-8, 10, 11]. Thus, the 2018 Physical Activity Guidelines for Americans and the 2010 American College of Sports Medicine (ACSM) exercise guidelines for cancer survivors recommends that adults avoid physical inactivity and exercise $\geq 150$ minutes per week and include strength training exercises on at least two days per week [12-14]. However, previous studies have found that rural cancer survivors are less likely to meet cancer-specific PA recommendations than urban cancer survivors $[4,15,16]$. Despite the overwhelming evidence supporting PA post-cancer diagnosis, more than half $(50.7 \%)$ of rural cancer survivors report being physically inactive, or not doing any leisure-time aerobic PA lasting at least 10 minutes, compared to only $38.7 \%$ of urban cancer survivors [4].

Although previous studies have explored the likelihood of meeting independent and combined exercise guidelines in cancer survivors [17-20], few studies have explored ruralurban differences in PA and self-rated health in cancer survivors. In a nationally representative sample of rural and urban cancer survivors in the U.S., Weaver and colleagues [4] reported that $30.5 \%$ of their sample was insufficiently active. Rogers and colleagues [21] found $27.0 \%$ of rural cancer survivors to be insufficiently active in their state cancer registry-based sample in Illinois, and Vallance and colleagues [22] reported that $65.3 \%$ were 
inactive in their state-based sample of rural and small town cancer survivors in Alberta, Canada. However, these studies focused on aerobic physical activity and did not capture muscle-strengthening activities.

In an effort to better design PA interventions for rural cancer survivors and to implement interventions within rural community settings, it is important to examine rural-urban differences in meeting PA recommendations and better understand which factors may be contributing to disparities in rural cancer survivors. Thus, the purposes of this study were to explore 1) rural-urban differences in meeting aerobic and muscle-strengthening PA recommendations and health status in cancer survivors in central Pennsylvania and 2) associations between PA and health status in rural and urban cancer survivors. This study is unique from others in that our study 1) included a representative sample of rural and urban cancer survivors in Pennsylvania, which is home to the fifth largest cancer survivor population in the U.S. [2]; 2) examined differences in meeting both aerobic and musclestrengthening physical activity whereas others only explored aerobic activity; and 3) explored associations between meeting individual recommendations and physical and mental health status.

\section{METHODS}

\section{Design, Sampling and Procedures}

We conducted a cross-sectional study with a population-based sample of cancer survivors in Pennsylvania, home to over 700,000 cancer survivors and the fifth largest cancer survivor population in the U.S. [2]. The Pennsylvania Cancer Registry was used to identify eligible and representative cancer survivors who 1) were $\geq 20$ years of age, 2) had received a breast, lung, colorectal, prostate, or gynecologic (cervical, endometrial, ovarian or uterine) cancer diagnosis between January 1, 2015 and December 31, 2016, and 3) were able to read, speak and write in English and respond to mailed surveys. A random sample of 2500 cancer survivors from 28 central Pennsylvania counties, 500 from each of five cancer sites, were mailed surveys. To ensure sufficient representation by race/ethnicity, we oversampled nonHispanic black and Hispanic by a factor of two. For cancers affecting both men and women (lung and colorectal cancers), we evenly sampled between men and women. Of those 28 counties, 18 are categorized as urban/metropolitan and 10 as rural/nonmetropolitan using the 2013 Rural/Urban Continuum Codes (RUCC) [23]. Participants in urban and rural counties were sampled relative to population size, and participants whose registry data were missing age, race/ethnicity, or zip code were excluded from this study. A National Death Index review was conducted before each mailing to avoid sending the survey to the homes of deceased cancer survivors. Figure 1 shows the flow of participants throughout the study.

Eligible participants were mailed questionnaires based on questions included in the Behavioral Risk Factor Surveillance System (BRFSS) from April-July 2017. Participants were initially sent a study recruitment letter with a brief description of the study and were instructed to opt-in or opt-out of the study by contacting the study team by phone, mail or email. After two weeks, those who did not opt-out were mailed a consent note and questionnaires with instructions to return in a self-addressed stamped envelope ( $1^{\text {st }}$ mailing). Four weeks later, those who had not returned completed questionnaires were re-sent 
materials ( $2^{\text {nd }}$ mailing). Those who had not returned their questionnaires four weeks after the second mailing were sent an additional, identical third mailing. Participants who optedout by phone, mail or email were no longer contacted. Participants who opted-in after the initial recruitment letter or returned completed questionnaires provided implied consent to participate in the study. All study procedures and materials were reviewed and approved by the Penn State College of Medicine's Institutional Review Board, and all participants provided informed implied consent.

\section{Measures}

Demographics and Rural-Urban Residence-Age, gender, cancer site and county of residence at diagnosis were extracted from the Pennsylvania Cancer Registry. Additional demographic information, including education and annual household income, was collected via self-report.

Rural-urban residence was based on county of residence at diagnosis, to which we applied the 2013 RUCC. The RUCC distinguish counties by their population size, degree of urbanization, and how adjacent they are to a metro area [23]. Each county in the U.S. is assigned one of nine codes, allowing for the assessment of trends related to population density and metropolitan influence. Urban counties are designated by codes 1-3 and include counties in metro areas with populations fewer than 250,000 to over one million. Nonmetro and rural counties are designated by codes 4-9 and include counties in nonmetro areas that may or may not be adjacent to a metro area with populations ranging from less than 2,500 to 20,000 or more [23].

Physical Activity-Self-reported leisure-time PA was assessed using questions based on the 2015 edition of the BRFSS [24]. Participants were asked if they participated in any PA or exercises during the past month. Those who responded 'yes' were asked to specify the type of activity, frequency per week or month, and duration. Participants were given the option to provide information for up to two activities or exercises and were asked to report how many times per week of month they performed muscle-strengthening activities [24]. Activities or exercises were coded and categorized as aerobic and moderate- or vigorous-intensity [25, 26]. Using the BRFSS scoring protocol [26], minutes per week of aerobic PA and times per week of muscle-strengthening activities were calculated. To maintain consistency with the 2018 Physical Activity Guidelines for Americans and the 2010 ACSM exercise guidelines for cancer survivors [12,13], which had not yet been updated at the time of this study, participants were categorized as meeting recommendations for 1) aerobic PA ( $\geq 150$ minutes of moderate-intensity or $\geq 75$ minutes of vigorous-intensity PA per week), 2) musclestrengthening activities ( $\geq 2$ times per week), 3) both aerobic and muscle-strengthening activities, or 4) neither aerobic nor muscle-strengthening activities.

Health Status-Health status was assessed using three items from the 2015 edition of the BRFSS [24]. Participants were asked to report the number of days within the past 30 days that their physical and mental health were not good and the number of days that their poor physical and/or mental health kept them from their usual activities. Participants who reported $<7$ days on which they experienced poor physical or mental health or $<7$ days on 
which their poor physical/mental health impeded their usual activities (e.g., self-care, work, recreation) were categorized as having good health compared to those who reported $\geq 7$ unhealthy days.

\section{Statistical Analysis}

Analyses were conducted in SPSS Version 24.0 (IBM SPSS Statistics, Armonk, NY), and statistical significance was set at $p<.05$. Independent samples t-tests and chi-square tests were used to assess rural-urban differences in demographics. We conducted a series of logistic regression analyses to assess rural-urban differences in meeting PA recommendations (neither vs. aerobic, muscle-strengthening, or both), adjusting for cancer type, gender, and income. Lastly, logistic regression models were used to explore the associations between meeting PA recommendations and dichotomized poor physical health days ( $\geq 7$ days vs. $<7$ days), poor mental health days ( $\geq 7$ days vs. $<7$ days), and poor physical or mental health days impeding activities ( $\geq 7$ days vs. $<7$ days). Regression analyses included age, cancer type (breast, lung, colorectal, prostate, or gynecologic), gender, and income ( $<\$ 35,00, \$ 35,000$ to $\$ 74,999$, or $\$ \$ 75,000$ per year), because they were associated with PA and rural-urban residence. Effect modification by rural-urban differences was assessed.

\section{RESULTS}

\section{Participant Characteristics}

Of the 2,463 cancer survivors mailed recruitment letters and questionnaires, 591 (24.0\%) returned completed questionnaires and participated in this study (Figure 1); the response rate did not significantly differ between rural (19.8\%) and urban (24.5\%) cancer survivors $\left(\chi^{2}=3.1, p=.078\right)$. Breast cancer survivors were most likely to return completed questionnaires, followed by gynecologic, prostate, and colorectal cancer survivors; lung cancer survivors were least likely to participate. Demographic characteristics, PA, and health status are summarized in Table 1 by rural-urban residence. Half (50.0\%) of rural cancer survivors reported being physically inactive (reported no leisure-time PA) compared to $35.2 \%$ of urban cancer survivors $\left(\chi^{2}=7.9, p=.020\right)$. Fewer rural cancer survivors met aerobic PA recommendations (17.1 vs. $33.5 \%$ ), but more rural cancer survivors reported meeting recommendations for muscle-strengthening activities ( 12.2 vs. $\left.5.6 \%, \chi^{2}=8.0, p=.047\right)$. There were no other significant differences in demographics or health status by rural-urban residence.

\section{Association between Rural-Urban Residence and Physical Activity}

The association between rural-urban residence and the likelihood of meeting PA recommendations is shown in Table 2. Urban cancer survivors were more than twice as likely to report meeting aerobic PA recommendations ( $\mathrm{OR}=2.6$, 95\% CI: 1.1-6.3) than rural cancer survivors in unadjusted (Model 1) and adjusted (Model 2) models. Adjusted models (Model 2) including age, cancer type, gender, and income revealed that lung cancer survivors were less likely to meet aerobic PA guidelines (OR=0.3, 95\% CI: 0.1-0.9) than those with other cancer types. Additionally, cancer survivors whose annual household income was less than $\$ 35,000$ were half as likely to meet aerobic PA recommendations 
( $\mathrm{OR}=0.5,95 \%$ CI: $0.3-0.8)$ than those who reported high income ( $\$ 75,000$ per year). There were no significant associations between rural-urban residence and meeting musclestrengthening PA guidelines, combined PA guidelines, or physical or mental health status.

\section{Associations between Physical Activity and Health Status}

Associations between meeting PA recommendations and health status, adjusted for rurality, age, cancer type, gender, and income, are shown in Table 3. Fully adjusted models (Model 3) showed that cancer survivors who reported meeting aerobic PA recommendations had nearly twice as high odds of reporting good physical health (OR=1.9, 95\% CI: 1.0-3.6) and odds 2.3 times higher to report good physical and mental health (OR=2.3, 95\% CI: 1.1-4.5) than cancer survivors who met neither aerobic nor muscle-strengthening recommendations. Additionally, lung cancer survivors ( $\mathrm{OR}=0.3,95 \% \mathrm{CI}$ : $0.1-0.8$ ) and cancer survivors reporting an annual household income less than $\$ 35,000$ ( $\mathrm{OR}=0.5,95 \% \mathrm{CI}$ : $0.3-0.9$ ) were less likely to report good physical health than those with other cancer types and high income, respectively. Rural-urban residence did not significantly moderate the effect of PA on health status in logistic regression models.

\section{DISCUSSION}

Although rural-urban differences in PA and health status have been described, previous studies did not examine differences in meeting aerobic and muscle-strengthening PA recommendations. This study extends those findings to highlight disparities in meeting aerobic and muscle-strengthening PA recommendations for cancer survivors and found that urban cancer survivors were two times more likely to meet aerobic PA recommendations than rural cancer survivors. Additionally, cancer survivors who reported meeting aerobic PA recommendations were more likely to report their health status as good. Given that rural cancer survivors are less likely to meet PA recommendations than their urban counterparts, these findings underscore the need for interventions to increase PA and improve health outcomes in rural cancer survivors in an effort to reduce cancer health disparities in this population.

Similar to previous studies, the majority of cancer survivors in this study reported not meeting PA recommendations [4, 21, 22]. We found the prevalence of leisure-time PA to be lower and the prevalence of physical inactivity to be higher among rural cancer survivors than urban cancer survivors in our study and similar to what has been previously reported in the general cancer survivor population in the U.S. [4, 27]. Further exploration of the likelihood of meeting aerobic and muscle-strengthening PA recommendations showed that rural cancer survivors were less likely to meet aerobic PA recommendations than urban cancer survivors, but not muscle-strengthening guidelines. This suggests that differences in aerobic PA may drive rural-urban differences in leisure-time PA in cancer survivors that have been reported previously. Additional research is needed to explore differences in physical activity by domain (e.g., leisure-time, work-related, domestic) and intensity (e.g., light, moderate, vigorous) to help inform future PA promotion efforts in rural cancer survivors. There has been a recent focus on distance- and home-based physical activity interventions in rural populations, which typically focus on exercises that can be easily implemented within 
the home, such as strength-training exercises [28]. Future interventions should test hybrid approaches to both reduce physical inactivity and increase aerobic PA, which may be a valuable starting point toward more comprehensive PA adoption and maintenance in rural cancer survivors.

Although we found no significant difference between rural and urban cancer survivors for health status, previous studies have shown substantial rural-urban differences in health status outcomes. A small rural sample size may have limited our power to detect statistically significant differences and limited our ability to complete stratified analyses by cancer type or socioeconomic status, which may help explain disparities in PA and health status [3, 29, 30]. However, it is important to highlight that $24.3 \%$ and $18.9 \%$ of cancer survivors in this study reported their physical and mental health, respectively, as 'not good' for one week or more in the past month, regardless of rural-urban residence. Additionally, nearly one-fifth $(19.6 \%)$ of rural cancer survivors reported that their poor physical or mental health impeded their usual activities for one week or more in the past month. These values are substantially higher than those previously reported by rural adults with no history of cancer and are concerning [31, 32].

Consistent with other studies among cancer survivors, we found a significant association between PA and health status [4, 33-38]. Our findings extend this work and showed that cancer survivors who reported meeting both aerobic and muscle-strengthening PA recommendations were more than twice as likely to report being in good health than those who did not meet PA recommendations. These findings further demonstrate the individual and complementary roles these activities play in improving health-related quality of life in rural and urban cancer survivors, and suggest that PA intensity and type are important for achieving both physical and mental health [33, 35, 37].

This study population was drawn from a representative sample of rural and urban cancer survivors in central Pennsylvania. A significant strength of this study is the inclusion of both aerobic and muscle-strengthening PA recommendations, consistent with the ACSM exercise guidelines for cancer survivors [12-14]. Previous studies have solely categorized cancer survivors as sufficiently active, insufficiently active, or inactive [4], which may not accurately capture rural-urban differences in meeting components of the PA guidelines. Given the recent update to the exercise guidelines for cancer survivors [14], additional work is needed to further explore adherence to the updated guidelines along with aerobic and muscle-strengthening PA recommendations, individually, among urban and rural cancer survivors.

Although this study makes a significant contribution to the existing literature on rural-urban differences in cancer survivors, it is not without limitations. First, this study included a small sample of cancer survivors who were classified as rural/non-metro using the 2013 RUCC codes [23]. Imbalances between groups may have impacted findings and limited our ability to draw conclusions about differences between rural and urban cancer survivors. However, post hoc sample size calculations demonstrated sufficient power (83.3\%) to detect an odds ratio of 2.6. Further research is needed to explore additional differences in physical activity and health status by rurality and cancer type. Additionally, although the RUCC codes 
account for population density and metro influence, they are assigned at the county-level and do not account for commuting areas or medical service areas, which may more accurately capture the heterogeneity in rural areas in Pennsylvania. Alternative definitions of rural, such as the Rural-Urban Commuting Area (RUCA) Codes [39] and the Center for Rural Pennsylvania's definition based on Pennsylvania-specific population density [40], were considered for this study. However, the RUCC codes have been used previously to explore rural-urban differences in cancer survivors and the general U.S. population $[1,4,31]$, lending to consistency and allowing for comparisons to previous studies. Additionally, the use of the RUCA codes did not change study findings. Thus, future studies should explore alternative definitions of rural/non-metro and potentially attempt to oversample rural cancer survivors, which may impact subsample sizes researchers should choose the most pragmatic measure to facilitate intervention implementation. Second, we relied on self-report PA and health status data. Although the over-reporting of PA is well-documented [41-43], there is no evidence to date documenting reporting differences between rural and urban cancer survivors. We relied on previously validated and reliable measures from the BRFSS to further reduce threats to internal validity and allow for comparisons with previous studies. However, these measures do not capture other types of PA, such as occupational or transportation-related activities, and may not be representative of the types of PA rural cancer survivors do. Future studies should include additional measures to assess PA done outside of leisure-time and should utilize wearable devices and sensors, such as accelerometers, to more objectively assess physical activity. Third, this study used a crosssectional design to explore associations between PA and health status. Therefore, we cannot determine directionality or causal pathways and whether PA leads to improvements in health status or vice versa. Lastly, this study included a state cancer registry-based sample of cancer survivors. Although this is the first study to our knowledge to report on PA and health status of cancer survivors in Pennsylvania, the use of a state cancer registry-based sample limits the generalizability of findings to rural and urban cancer survivors in other geographic regions, such as frontier populations. Additionally, this study was limited to breast, lung, colorectal, prostate, and gynecological cancer survivors at least 20 years of age or older, whom we have stronger evidence supporting the benefits of physical activity [14]. Although sampling was restricted to these cancer sites to ensure sufficient representation by cancer type and to avoid uneven sampling, particularly in rural counties, this limits the generalizability of findings to other cancer types and age groups. Lastly, we were unable to accurately determine treatment status within the cancer registry. Therefore, it is possible that the study sample included participants who were receiving treatment as well as those who were post-treatment or in remission. Additional research is needed to explore rural-urban differences in physical activity and health status by time since treatment.

\section{CONCLUSIONS}

This study is both innovative and timely, given the recent focus on rural cancer control by the American Association for Cancer Research, the American Cancer Society, the American Society of Clinical Oncology, and the National Cancer Institute [3, 5]. Results from this study contribute to the growing literature documenting rural-urban health disparities in cancer survivors and document persistent rural-urban differences in the likelihood of 
meeting PA recommendations in cancer survivors. Given the strong association between aerobic PA and health status in cancer survivors, these findings suggest the need for targeted behavior change interventions to increase aerobic PA, specifically, in rural cancer survivors, which may contribute to improvements in health-related quality of life and aid efforts to reduce cancer health disparities. Additional research is needed to further explore rural cancer survivors' preferences for PA and barriers to meeting aerobic and muscle-strengthening PA recommendations to inform the design and adaptation of evidence-based PA interventions for rural cancer survivors.

\section{Acknowledgements}

The authors appreciate the cancer survivors who responded to surveys and participated in this study. We also acknowledge the assistance of the Community Sciences and Health Outcomes Shared Resource of the Penn State Cancer Institute in generating the sampling strategy for this study. These data were supplied by the Bureau of Health Statistics \& Registries, Pennsylvania Department of Health, Harrisburg, Pennsylvania. The Pennsylvania Department of Health specifically disclaims responsibility for any analyses, interpretations or conclusions.

Funding sources: Scherezade Mama is supported by a career development award from the National Institutes of Health National Cancer Institute (K07 CA22335-A101).

\section{References}

1. Weaver KE, Geiger AM, Lu L, Case LD. Rural-urban disparities in health status among US cancer survivors. Cancer. 2013;119(5):1050-7. [PubMed: 23096263]

2. American Cancer Society. Cancer Treatment \& Survivorship Facts \& Figures 2016-2017. Atlanta, GA: American Cancer Society; 2016.

3. Blake KD, Moss JL, Gaysynsky A, Srinivasan S, Croyle RT. Making the Case for Investment in Rural Cancer Control: An Analysis of Rural Cancer Incidence, Mortality, and Funding Trends. Cancer Epidemiol Biomarkers Prev. 2017;26(7):992-7. [PubMed: 28600296]

4. Weaver KE, Palmer N, Lu L, Case LD, Geiger AM. Rural-urban differences in health behaviors and implications for health status among US cancer survivors. Cancer Causes Control. 2013;24(8):1481-90. [PubMed: 23677333]

5. Polite BN, Adams-Campbell LL, Brawley OW, Bickell N, Carethers JM, Flowers CR, et al. Charting the future of cancer health disparities research: A position statement from the American Association for Cancer Research, the American Cancer Society, the American Society of Clinical Oncology, and the National Cancer Institute. CA Cancer J Clin. 2017.

6. Lahart IM, Metsios GS, Nevill AM, Carmichael AR. Physical activity, risk of death and recurrence in breast cancer survivors: A systematic review and meta-analysis of epidemiological studies. Acta Oncol. 2015;54(5):635-54. [PubMed: 25752971]

7. Doyle C, Kushi LH, Byers T, Courneya KS, Demark-Wahnefried W, Grant B, et al. Nutrition and physical activity during and after cancer treatment: an American Cancer Society guide for informed choices. CA Cancer J Clin. 2006;56(6):323-53. [PubMed: 17135691]

8. Rock CL, Doyle C, Demark-Wahnefried W, Meyerhardt J, Courneya KS, Schwartz AL, et al. Nutrition and physical activity guidelines for cancer survivors. CA Cancer J Clin. 2012;62(4):24374. [PubMed: 22539238]

9. Cormie P, Zopf EM, Zhang X, Schmitz KH. The Impact of Exercise on Cancer Mortality, Recurrence, and Treatment-Related Adverse Effects. Epidemiol Rev. 2017;39(1):71-92. [PubMed: 28453622]

10. Mishra SI, Scherer RW, Geigle PM, Berlanstein DR, Topaloglu O, Gotay CC, et al. Exercise interventions on health-related quality of life for cancer survivors. Cochrane Database Syst Rev. 2012(8):CD007566. [PubMed: 22895961]

11. Mishra SI, Scherer RW, Snyder C, Geigle PM, Berlanstein DR, Topaloglu O. Exercise interventions on health-related quality of life for people with cancer during active treatment. Cochrane Database Syst Rev. 2012(8):CD008465. [PubMed: 22895974] 
12. Schmitz KH, Courneya KS, Matthews C, Demark-Wahnefried W, Galvao DA, Pinto BM, et al. American College of Sports Medicine roundtable on exercise guidelines for cancer survivors. Med Sci Sports Exerc. 2010;42(7):1409-26. [PubMed: 20559064]

13. U.S. Department of Health and Human Services. Physical Activity Guidelines for Americans, 2nd edition. Washington, DC: U.S. Department of Health and Human Services; 2018.

14. Campbell KL, Winters-Stone KM, Wiskemann J, May AM, Schwartz AL, Courneya KS, et al. Exercise Guidelines for Cancer Survivors: Consensus Statement from International Multidisciplinary Roundtable. Med Sci Sports Exerc. 2019;51(11):2375-90. [PubMed: 31626055]

15. Irwin ML, McTiernan A, Bernstein L, Gilliland FD, Baumgartner R, Baumgartner K, et al. Physical activity levels among breast cancer survivors. Med Sci Sports Exerc. 2004;36(9):148491. [PubMed: 15354027]

16. Paskett ED. Breast Cancer Among Special Populations: Disparities in Care Across the Cancer Control Continuum. Adv Exp Med Biol. 2015;862:39-52. [PubMed: 26059928]

17. Vallerand JR, Rhodes RE, Walker GJ, Courneya KS. Correlates of meeting the combined and independent aerobic and strength exercise guidelines in hematologic cancer survivors. Int J Behav Nutr Phys Act. 2017;14(1):44. [PubMed: 28351397]

18. Trinh L, Strom DA, Wong JN, Courneya KS. Modality-specific exercise guidelines and quality of life in kidney cancer survivors: A cross-sectional study. Psychooncology. 2018;27(10):2419-26. [PubMed: 30048023]

19. Tabaczynski A, Strom DA, Wong JN, McAuley E, Larsen K, Faulkner GE, et al. Demographic, medical, social-cognitive, and environmental correlates of meeting independent and combined physical activity guidelines in kidney cancer survivors. Support Care Cancer. 2020;28(1):43-54. [PubMed: 30980259]

20. Crawford JJ, Holt NL, Vallance JK, Courneya KS. A new paradigm for examining the correlates of aerobic, strength, and combined exercise: an application to gynecologic cancer survivors. Support Care Cancer. 2016;24(8):3533-41. [PubMed: 27021390]

21. Rogers LQ, Markwell SJ, Verhulst S, McAuley E, Courneya KS. Rural breast cancer survivors: exercise preferences and their determinants. Psychooncology. 2009;18(4):412-21. [PubMed: 19241491]

22. Vallance J, Lavallee C, Culos-Reed N, Trudeau M. Rural and small town breast cancer survivors' preferences for physical activity. Int J Behav Med. 2013;20(4):522-8. [PubMed: 22992864]

23. United States Department of Agriculture (USDA). Rural-Urban Continuum Codes: USDA Economic Research Service; [updated October 12, 2016 Available from: https://www.ers.usda.gov/ data-products/rural-urban-continuum-codes/.

24. Centers for Disease Control and Prevention (CDC). BRFSS Questions: Behavioral Risk Factor Surveilliance System (BRFSS); 2017 [updated August 25, 2017 Available from: https:// www.cdc.gov/brfss/questionnaires/index.htm.

25. Ainsworth BE, Haskell WL, Herrmann SD, Meckes N, Bassett DR Jr., Tudor-Locke C, et al. 2011 Compendium of Physical Activities: a second update of codes and MET values. Med Sci Sports Exerc. 2011;43(8):1575-81. [PubMed: 21681120]

26. Centers for Disease Control and Prevention. A Data Users Guide to the BRFSS Physical Activity Questions: How to Assess the 2008 Physical Activity Guidelines for Americans: Centers for Disease Control and Prevention; [Available from: https://www.cdc.gov/brfss/pdf/PA \%20RotatingCore_BRFSSGuide_508Comp_07252013FINAL.pdf.

27. Smith WA, Nolan VG, Robison LL, Hudson MM, Ness KK. Physical activity among cancer survivors and those with no history of cancer- a report from the National Health and Nutrition Examination Survey 2003-2006. Am J Transl Res. 2011;3(4):342-50. [PubMed: 21904654]

28. Vallance J, Basen-Engquist K, Rogers LQ, Alfano C. Distance-based physical activity trials for cancer survivors: Current evidence, novel interventions, and future directions. Ann Behav Med. 2019;53(Suppl 1):S195-S7.

29. Cohen SA, Cook SK, Kelley L, Foutz JD, Sando TA. A Closer Look at Rural-Urban Health Disparities: Associations Between Obesity and Rurality Vary by Geospatial and Sociodemographic Factors. J Rural Health. 2017;33(2):167-79. [PubMed: 27557442] 
30. Long AS, Hanlon AL, Pellegrin KL. Socioeconomic variables explain rural disparities in US mortality rates: Implications for rural health research and policy. SSM Popul Health. 2018;6:72-4. [PubMed: 30225336]

31. James CV, Moonesinghe R, Wilson-Frederick SM, Hall JE, Penman-Aguilar A, Bouye K. Racial/ Ethnic Health Disparities Among Rural Adults - United States, 2012-2015. MMWR Surveill Summ. 2017;66(23):1-9.

32. Dwyer-Lindgren L, Mackenbach JP, van Lenthe FJ, Mokdad AH. Self-reported general health, physical distress, mental distress, and activity limitation by US county, 1995-2012. Popul Health Metr. 2017;15:16.

33. Garcia DO, Thomson CA. Physical activity and cancer survivorship. Nutr Clin Pract. 2014;29(6):768-79. [PubMed: 25335787]

34. Phillips SM, McAuley E. Physical activity and quality of life in breast cancer survivors: the role of self-efficacy and health status. Psychooncology. 2014;23(1):27-34. [PubMed: 24003002]

35. Lee HY, Kim J, Merighi JR. Physical Activity and Self-Rated Health Status Among Older Adult Cancer Survivors: Does Intensity of Activity Play a Role? Oncol Nurs Forum. 2015;42(6):614-24. [PubMed: 26488831]

36. Smith AW, Alfano CM, Reeve BB, Irwin ML, Bernstein L, Baumgartner K, et al. Race/ethnicity, physical activity, and quality of life in breast cancer survivors. Cancer Epidemiol Biomarkers Prev. 2009;18(2):656-63. [PubMed: 19190157]

37. Courneya KS, Friedenreich CM. Physical exercise and quality of life following cancer diagnosis: a literature review. Ann Behav Med. 1999;21(2):171-9. [PubMed: 10499138]

38. Blanchard CM, Courneya KS, Stein K, American Cancer Society's SCS, II. Cancer survivors' adherence to lifestyle behavior recommendations and associations with health-related quality of life: results from the American Cancer Society's SCS-II. J Clin Oncol. 2008;26(13):2198-204. [PubMed: 18445845]

39. United States Department of Agriculture (USDA). Rural-Urban Commuting Area Codes: USDA Economic Research Service; [updated October 12, 2016; cited 2018. Available from: https:// www.ers.usda.gov/data-products/rural-urban-commuting-area-codes.aspx.

40. Rural Urban Definitions [Internet]. 2014 Available from: http://www.rural.palegislature.us/ demographics_rural_urban.html.

41. Lee RE, Mama SK, Medina AV, Reese-Smith JY, Banda JA, Layne CS, et al. Multiple measures of physical activity, dietary habits and weight status in African American and Hispanic or Latina women. J Community Health. 2011;36(6):1011-23. [PubMed: 21519867]

42. Mama SK, Leach HJ, Soltero EG, Lee RE. Improved Physical Activity Screening Enhances Intervention Effectiveness in Ethnic Minority Women: A Longitudinal Study. Health Promot Pract. 2016.

43. Pietilainen KH, Korkeila M, Bogl LH, Westerterp KR, Yki-Jarvinen H, Kaprio J, et al. Inaccuracies in food and physical activity diaries of obese subjects: complementary evidence from doubly labeled water and co-twin assessments. Int J Obes (Lond). 2010;34(3):437-45. [PubMed: 20010905] 


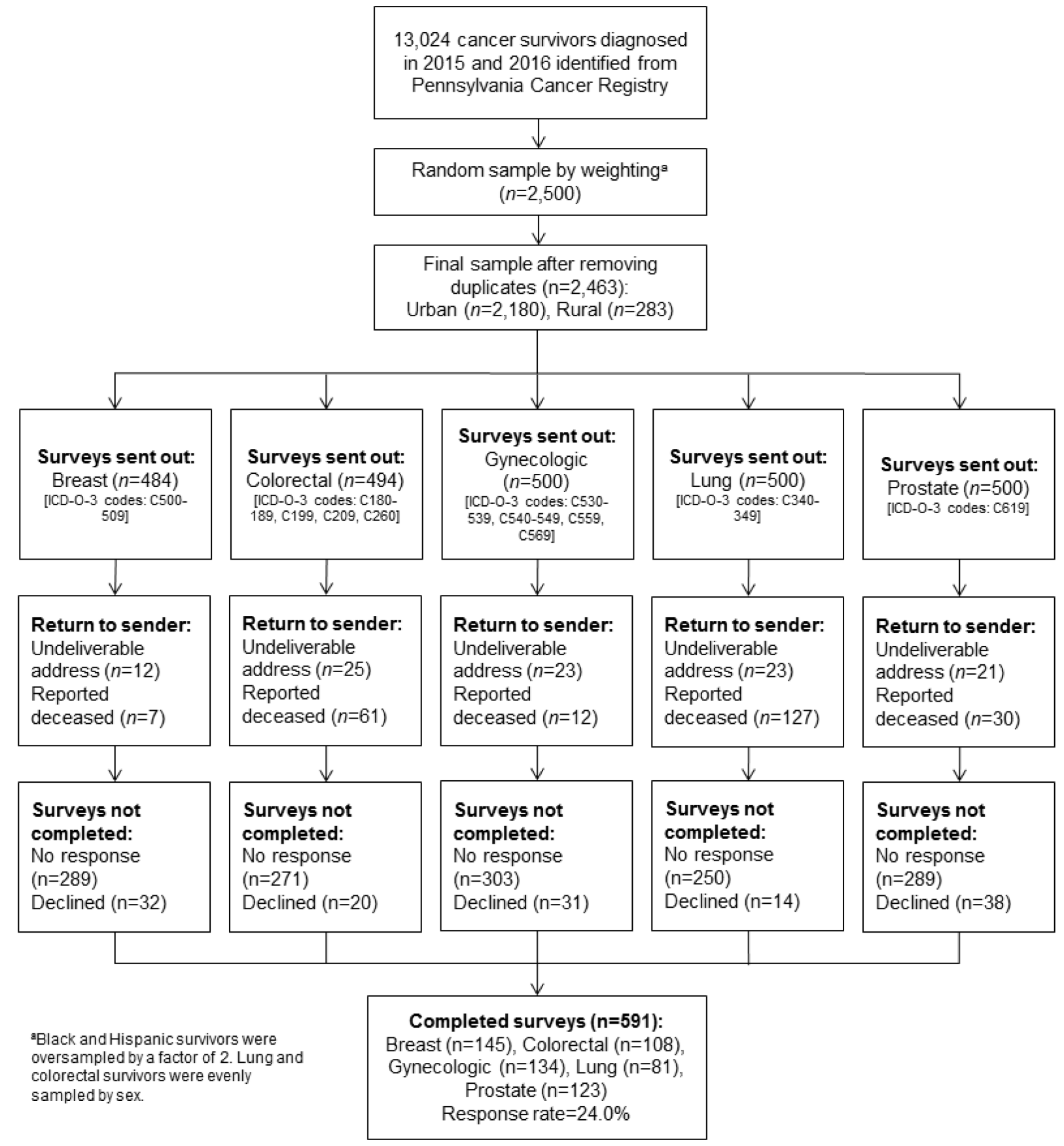

Figure 1.

Flow of participants throughout the study 
Table 1.

Participant characteristics by rural-urban residence

\begin{tabular}{|c|c|c|c|c|}
\hline & Rural (n=56) & Urban $(n=535)$ & Total $(N=591)$ & $p$ \\
\hline Age, years $(M \pm S D)$ & $66.7 \pm 11.5$ & $65.8 \pm 11.6$ & $65.9 \pm 11.6$ & .534 \\
\hline BMI, $\mathrm{kg} / \mathrm{m}^{2}(M \pm S D)$ & $31.0 \pm 9.1$ & $31.1 \pm 8.2$ & $31.1 \pm 8.3$ & .886 \\
\hline \multicolumn{5}{|l|}{$\operatorname{Sex}(N, \%)$} \\
\hline Male & $24,42.9$ & $192,35.9$ & $216,36.5$ & \multirow[t]{2}{*}{.303} \\
\hline Female & $32,57.1$ & $343,64.1$ & $375,63.5$ & \\
\hline \multicolumn{4}{|l|}{ Marital Status $(N, \%)$} & \multirow[t]{3}{*}{.976} \\
\hline Married/Living with Partner & $38,69.1$ & $361,68.9$ & $399,68.9$ & \\
\hline Not Married & $17,30.9$ & $163,31.1$ & $180,31.1$ & \\
\hline Hispanic $(N, \%)$ & $1,1.9$ & $19,3.7$ & $20,3.5$ & $.710^{*}$ \\
\hline \multicolumn{4}{|l|}{$\operatorname{Race}(N, \%)$} & \multirow[t]{4}{*}{.306} \\
\hline White & $53,96.4$ & $474,91.9$ & $527,92.3$ & \\
\hline Black or African American & $0,0.0$ & $21,4.1$ & $21,3.7$ & \\
\hline Other & $2,3.6$ & $21,4.1$ & $23,4.0$ & \\
\hline \multicolumn{4}{|l|}{ Education $(N, \%)$} & \multirow[t]{4}{*}{.340} \\
\hline$\leq$ High school or GED & $28,50.0$ & $210,39.8$ & $238,40.8$ & \\
\hline Some college & $12,21.4$ & $136,25.8$ & $148,25.4$ & \\
\hline$\geq 4$-year college degree & $16,28.6$ & $181,34.3$ & $197,33.8$ & \\
\hline \multicolumn{4}{|l|}{ Annual Income $(N, \%)$} & \multirow[t]{4}{*}{.130} \\
\hline$<\$ 35,00$ & $22,44.9$ & $164,37.2$ & $186,38.0$ & \\
\hline$\$ 35,000$ to $\$ 74,999$ & $18,36.7$ & $134,30.4$ & $152,31.0$ & \\
\hline$\geq \$ 75,000$ & $9,18.4$ & $143,32.4$ & $152,31.0$ & \\
\hline \multicolumn{4}{|l|}{ Employment status $(N, \%)$} & \multirow[t]{4}{*}{.075} \\
\hline Employed & $5,16.1$ & $119,36.1$ & $124,34.3$ & \\
\hline Unemployed & $4,12.9$ & $39,11.8$ & $43,11.9$ & \\
\hline Retired & $22,71.0$ & $172,52.1$ & $194,53.7$ & \\
\hline \multicolumn{4}{|l|}{ Cancer Type $(N, \%)$} & \multirow[t]{6}{*}{.345} \\
\hline Breast & $8,14.3$ & $137,25.6$ & $145,24.5$ & \\
\hline Lung & $11,19.6$ & $70,13.1$ & $81,13.7$ & \\
\hline Colorectal & $11,19.6$ & $97,18.1$ & $108,18.3$ & \\
\hline Prostate & $13,23.2$ & $110,20.6$ & $123,20.8$ & \\
\hline Gynecologic & $13,23.2$ & $121,22.6$ & $134,22.7$ & \\
\hline Meeting Physical Activity & & & & .047 \\
\hline \multicolumn{5}{|l|}{ Recommendations $(N, \%)$} \\
\hline Neither & $23,56.1$ & $172,41.7$ & $195,43.0$ & \\
\hline Aerobic & $7,17.1$ & $138,33.5$ & $145,32.0$ & \\
\hline Muscle-strengthening & $5,12.2$ & $23,5.6$ & $28,6.2$ & \\
\hline Both & $6,14.6$ & $79,19.2$ & $85,18.8$ & \\
\hline \multicolumn{5}{|c|}{ Unhealthy Days in Past Month $(N, \%)$} \\
\hline Poor physical health $\geq 7$ days & $13,28.9$ & $111,23.9$ & $124,24.3$ & .454 \\
\hline
\end{tabular}




\begin{tabular}{lllll}
\hline & Rural $(\boldsymbol{n = 5 6})$ & Urban $(\boldsymbol{n = 5 3 5})$ & Total $(\boldsymbol{N = 5 9 1})$ & $\boldsymbol{p}$ \\
\hline Poor mental health $\geq 7$ days & $5,10.6$ & $95,19.8$ & $100,18.9$ & .128 \\
Poor physical or mental health impeded usual activities $\geq 7$ days & $10,19.6$ & $81,16.1$ & $91,16.5$ & .524 \\
\hline
\end{tabular}

* Fisher's exact 


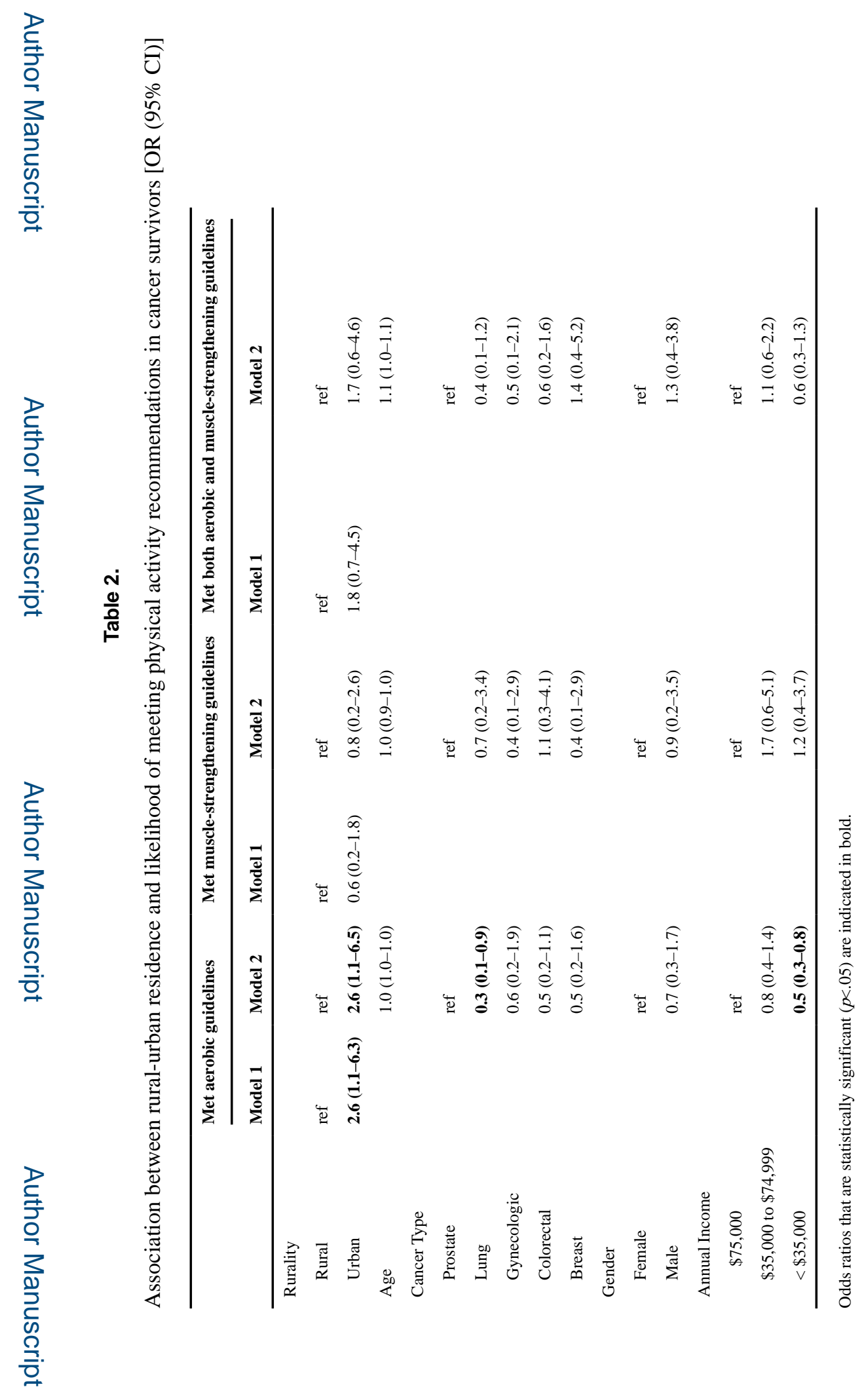

Support Care Cancer. Author manuscript; available in PMC 2021 October 01. 


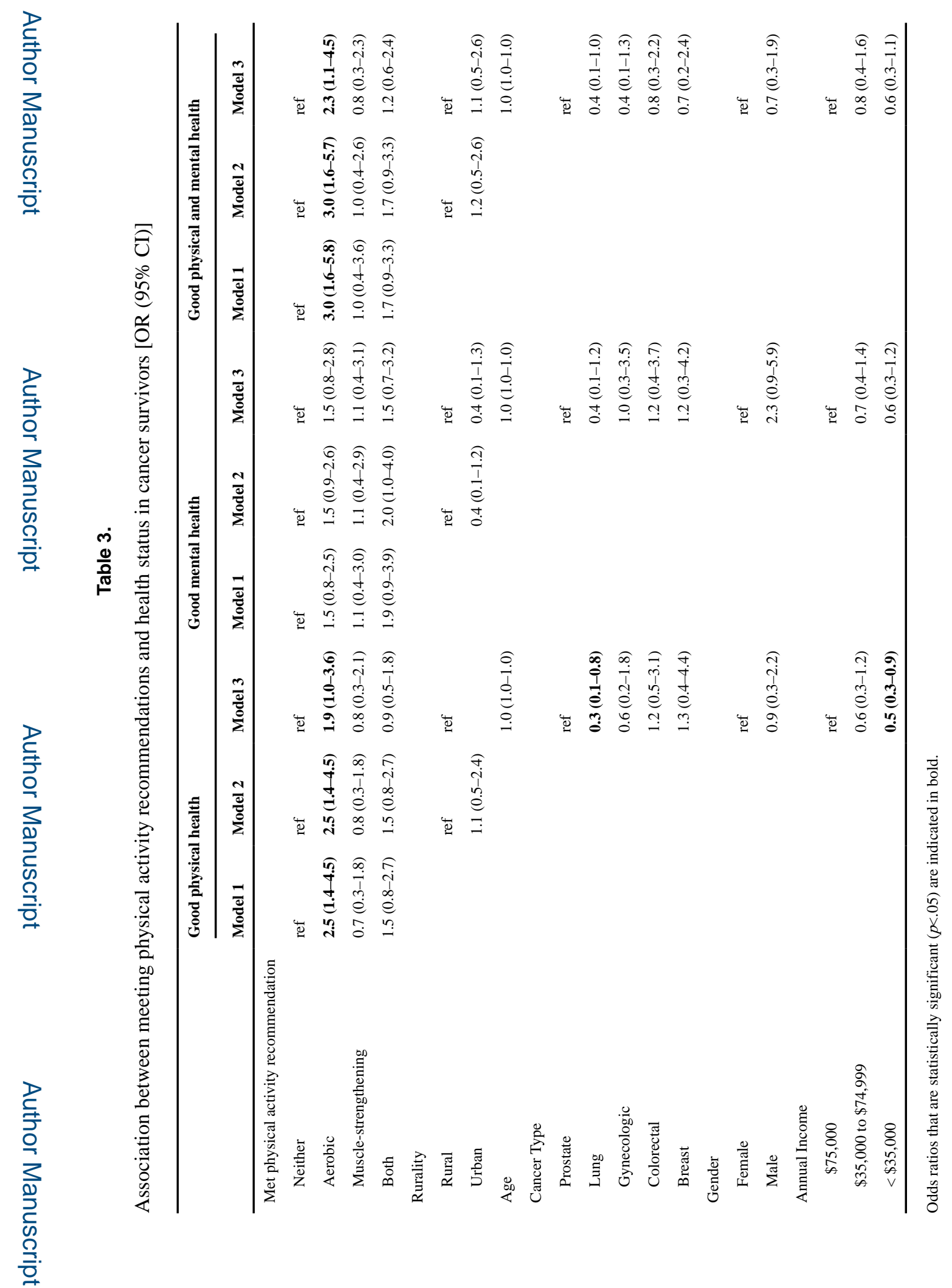

Support Care Cancer. Author manuscript; available in PMC 2021 October 01. 